\title{
DYNAMICAL THEORY OF DENSE GROUPS OF GALAXIES
}

\author{
Gary A. Mamon \\ DAEC, Observatoire de Meudon \\ Meudon, France
}

It is well known that galaxies associate in groups and clusters. Perhaps $40 \%$ of all galaxies are found in groups of 4 to 20 galaxies (e.g., Tully 1987). Although most groups appear to be so loose that the galaxy interactions within them ought to be insignificant, the apparently densest groups, known as compact groups appear so dense when seen in projection onto the plane of the sky that their members often overlap. These groups thus appear as dense as the cores of rich clusters. The most popular catalog of compact groups, compiled by Hickson (1982), includes isolation among its selection critera. Therefore, in comparison with the cores of rich clusters, Hickson's compact groups (hereafter, HCGs) appear to be the densest isolated regions in the Universe (in galaxies per unit volume), and thus provide in principle a clean laboratory for studying the competition of very strong gravitational interactions. The $\$ 64,000$ question here is then: Are compact groups really bound systems as dense as they appear? If dense groups indeed exist, then one expects that each of the dynamical processes leading to the interaction of their member galaxies should be greatly enhanced. This leads us to the questions: How stable are dense groups? How do they form? And the related question, fascinating to any theorist: What dynamical processes predominate in dense groups of galaxies?. If HCGs are not bound dense systems, but instead 1D chance alignments (Mamon 1986, 1987; Walke \& Mamon 1989) or 3D transient cores (Rose 1979) within larger looser systems of galaxies, then the relevant question is: How frequent are chance configurations within loose groups? In this review, I will answer these last four questions after comparing in some detail the methods used and the results obtained in the different studies of dense groups, while in the accompanying contribution, I will attempt to reconcile the recent observations of galaxy interactions in HCGs with a negative answer to the first question.

\section{Constructing dense groups}

At first approximation, a group of galaxies is a collection of galaxies, each of which is a collection of point masses. In fact, one must add to this model a component for the dark matter that occupies perhaps $90 \%$ of the total mass, and here lies perhaps the greatest uncertainty in the modelling, whether on paper or numerical. In practice, either one assumes that the dark matter lies mainly in individual halos around galaxies, or else one has it stretch over an intergalactic background (hereafter, IGB). As for the galaxies, they can be spiral, with disk, bulge and dark halo, or elliptical (with in principle a dark halo too).

Studies of dense groups have always been numerical in nature (see the following section). The simulations published in the litterature can be subdivided into two main categories. In "self-consistent" simulations, authors have attempted to reproduce the galaxies in the groups with as many particles as computationally feasible to run at least one simulation. On the 
other hand, some authors have sacrificed accuracy by representing each galaxy as a single particle (Mamon 1987 models the IGB in the same way) in order to achieve computational speed. In this second scheme the dynamical processes must be explicitly included in the computational code, hence I coin the name "explicit-physics" method. Alternatively one could call the two the "slow and clean" and the "quick and dirty" methods. In Table 1 below, I list the pros and cons of each of these two methods.

Table 1: Two opposite philosophies with their advantages

\begin{tabular}{ll}
\hline \hline $\begin{array}{l}\text { SELF-CONSISTENT } \\
\text { (many particles per galaxy) }\end{array}$ & $\begin{array}{l}\text { EXPLICIT-PHYSICS } \\
\text { (one particle per galaxy) }\end{array}$ \\
\hline Confidence in results & $\begin{array}{l}\text { Explore parameter space } \\
\text { Not only virialized initial conditions }\end{array}$ \\
$\begin{array}{l}\text { Accurate description of close encounters } \\
\text { Simpler to code }\end{array}$ & Compare dynamical processes \\
Project pretty movies & \\
\hline
\end{tabular}

Neither of the two methods is intrinsically superior to the other, but rather complementary. For example, if one is to understand the evolution of dense groups, one needs to study groups with different initial densities, membership, dark matter location, etc. The parameter space thus turns out to be quite large. Moreover, to build statistical sets of simulated evolving groups, one needs a quick method. What is the need for so many simulations? First, one would like to know if a small fraction (say $5 \%$ ) of dense groups can survive as such for long periods of time. Second, one would like to perform a variety of statistical tests to both the simulated groups and the observed HCGs. For these reasons, a set of roughly 1000 simulations of dense groups seems required if one is to understand the evolution of dense groups, and at this point these are done by the less-accurate explicit-physics method. An interesting feature of the explicit-physics method lies in the fact that the importance of each dynamical process can be gauged by simulating groups with the dynamical process artificially turned off and comparing the results with those using the standard physics. However one should also stress that, simulations of the self-consistent type are required to give confidence to the builders of the explicit-physics simulations of the meaningfulness of their results. In Table 2 are listed the self-consistent studies of dense groups.

Table 2: Self-consistent studies

\begin{tabular}{lccccccc}
\hline \hline Author(s) & $N$ & $n$ & Galaxies & IGM & Init. Conds. & IMF & $\mathcal{N}$ \\
\hline Carnevali et al. (81) & $10-20$ & 20 & Sph & 0 & Vir & $\delta$ & 3 \\
Ishizawa et al. (83) & 10 & 100 & Sph & 0 & Coll & $\delta$ & 1 \\
Cavaliere et al. (83) & $10-20$ & $20 ?$ & Sph & $0.5-0.9$ & Vir, Exp & $\delta$, Schechter & $5 ?$ \\
Barnes (85) & $5-10$ & $75-300$ & Sph & $0-0.75$ & Vir, Exp, Coll & $\delta$ & 27 \\
Ishizawa (86) & $10-50$ & 100 & Sph & 0 & Coll, Vir & $\delta$ & 7 \\
Barnes (89) & 6 & $8 \mathrm{k}-16 \mathrm{k}$ & $\mathrm{D}+\mathrm{B}+\mathrm{H}$ & 0 & Vir & $1,1,1,1,2,2$ & 1 \\
\hline
\end{tabular}

NOTES: $N$ is the number of galaxies per group, $n$ the number of particles per galaxy, "Galaxies" refers to the galaxy model (Sph $\rightarrow$ spherical, and D+B $+\mathrm{H} \rightarrow$ disk+bulge+halo), "IGM" is the fraction of intergalactic mass, "Init. Conds." refers to the initial conditions (Vir $\rightarrow$ viri- 
alized, Coll $\rightarrow$ collapsing, and Exp $\rightarrow$ expanding), "IMF" refers to the initial mass function $(\delta \rightarrow$ equal masses), $\mathcal{N}$ is the number of simulations run.

\section{Dynamical processes}

The one physical mechanism at work in dense groups is classical gravitation, i.e., the direct gravitational attraction between galaxies and between galaxies and the IGB, dynamical friction of galaxies against the IGB and against the stars of other galaxies, tidal processes, and mergers. I analyze each in turn.

Galaxy motions are set by their gravitational attraction to one another, as well as to the IGB. Whatever the type of simulation method used, one has to modify the potential carried by a particle at close range. For the self-consistent simulations, this modification is required to avoid a spurrious rapid relaxation caused by close encounters. On the other hand, for the explicit-physics simulations, this potential softening is done to mimic the extended sizes of galaxies. In my thesis (Mamon 1985), I've shown that, for realistic spherical galaxies of different masses and density profiles, the standard softening $\phi(r)=-G m_{1} m_{2} /\left(r^{2}+\epsilon^{2}\right)^{1 / 2}$ produces more accurate gradients than any $\gamma \neq 2$ in the more general formula $\phi(r)=$ $-G m_{1} m_{2} /\left(r^{\gamma}+\epsilon^{\gamma}\right)^{1 / \gamma}$. I also showed that the softening length $\epsilon$ is given to typically $25 \%$ accuracy by the rms of the half-mass radii of the two objects.

If the IGB is treated as a smooth medium (as in the explicit-physics methods), one needs to add the collective of effect of the encounters of galaxies with the much less massive particles that make up the IGB (e.g., black holes, brown dwarfs, or cold dark matter particles), known as dynamical friction. The analogous dynamical friction of the galaxies by the stars of the galaxies it encounters is implicit in any $N$-body explicit-physics or self-consistent method; and the latter method of course also includes implicitly the dynamical friction against the IGB, modelled as a collection of particles.

In dense environments, tidal effects are strong enough to significantly alter the member galaxies. Tidal processes come in two sorts: collisional tides caused by the passage of another galaxy, and the global IGB tide due to the non-uniform potential gradient of the IGB. By definition, tides cause various parts of a test galaxy to be subject to different accelerations. The galaxy is thus stretched and it's morphology can become quite disturbed, and can lose mass as some of its stars are accelerated beyond their escape velocity. Moreover, the stretching of the galaxy implies that energy is being imparted from the orbital motion of a colliding pair into their internal motions. With self-consistent simulations, collisional tides are implicit. As tidal processes cause the galaxies to shed stars, the IGB grows, and IGB tides are thus also implicit in this type of simulation. In explicit-physics simulations, one must include these tidal processes by hand, which is not always easy given the little that is understood about tides. More precisely, one must include a mass-loss, an internal energy-gain, a loss of orbital energy (often called tidal friction), hence a reduced galaxy velocity, and if the modeller has put some structure into his galaxies, he must know how to alter it. This is usually done by analysing the results of self-consistent two-body encounters usually done by others. The situation is all the more complicated that these parameter changes are a function of the internal orbits in the galaxies (Dekel et al. 1980) and of the galaxy orbits around the IGB (Mamon 1987).

An extreme case of the combination of dynamical friction of a galaxy against stars from another nearby galaxy, and collisional tides due to this encounter causes such a great loss of 
orbital energy that an unbound pair can become bound, and even worse, a bound pair can merge. Once again, this event occurs naturally in self-consistent simulations, but has to be mimicked explicitly in the explicit-physics type of calculation. And similarly to the case of non-merging encounters, one must specify for the larger ("cannibal") galaxy its new mass (some of the merging mass escapes to the IGB), internal and orbital energies, and structure.

In Table 3 below, I list the main ingredients of all the group and cluster simulations known to me that included at least tides or mergers. Note that the physics is the same for groups and clusters, which explains why cluster simulations are present in this table.

Table 3: Explicit-physics studies

\begin{tabular}{|c|c|c|c|c|c|c|c|c|c|c|}
\hline Author(s) & Meth & $N$ & Soft & IGB & ICs & IMF & $\mathrm{DF}$ & Merg & Tides & $\mathcal{N}$ \\
\hline Richstone (76) & QA & NR & NR & No & V & $\delta, 0$ & NR & No & $\mathrm{C}$ & 5 \\
\hline Jones \& Efstathiou (79) & $\mathbf{N}$ & 500 & Fix & No & $\mathbf{E}$ & $\delta$ & NR & Yes & No & 4 \\
\hline Roos \& Norman (79) & $\mathrm{N}$ & $10-60$ & Fix & No & $\vec{V}$ & $\delta$ & NR & Yes & $\mathrm{C}$ & 4 \\
\hline Aarseth \& Fall (80) & $\mathrm{N}$ & 1000 & $r_{1 / 2}$ & No & $\mathbf{E}$ & $\delta, \mathrm{O}$ & NR & Yes & No & 6 \\
\hline Cooper \& Miller (81) & $\mathbf{N}$ & 100 & Fix & No & V & $\delta$ & NR & Yes & No & 3 \\
\hline Roos (81) & $\mathrm{N}$ & 400 & $r$ & No & $\mathbf{E}$ & $\delta$ & NR & Yes & $\mathrm{C}$ & 14 \\
\hline Roos \& Áarseth (82) & $\mathrm{N}$ & $350-700$ & $\boldsymbol{r}$ & No & $\mathrm{E}$ & $\mathbf{S}$ & NR & Yes & $\mathrm{C}$ & 5 \\
\hline Schneider \& Gunn (82) & $\mathrm{N}$ & 1 & NR & Yes & Circ & NR & Yes & NR & IGB & 3 \\
\hline Miller (83) & 3 & $100 ?$ & NR & Yes & $\mathrm{E}, \mathrm{V}$ & $\delta, S$ & No & Yes & $\mathrm{C}$ & 18 \\
\hline Merritt (83) & QA & NR & NR & Yes & $\mathrm{V}$ & $\mathrm{S}$ & No & No & $\mathrm{C}$ & 3 \\
\hline Richstone \& Malumuth (83) & 3 & $100-1 k$ & NR & Yes & V & $\delta, S$ & Yes & Yes & $\mathrm{C}$ & 41 \\
\hline Malumuth \& Richstone (84) & 3 & $100-500$ & NR & Yes & V & $\dot{S}$ & Yes & Yes & $\mathrm{C}$ & 30 \\
\hline Saarinen \& Valtonen (85) & $\mathrm{N}$ & 100 & Fix & No & $\mathrm{C}, \mathrm{V}$ & 0 & NR & Yes & No & 48 \\
\hline Mamon (87) & $\mathrm{N}$ & $5-20$ & $r_{1 / 2}$ & Yes & $\mathrm{V}$ & $\delta, S$ & Yes & Yes & $\mathrm{C}+\mathrm{IGB}$ & $>1 \mathrm{k}$ \\
\hline Navarro et al. (87) & $\mathrm{N}$ & $50-100$ & $r_{1 / 2}$ & Yes & $\mathrm{V}, \mathrm{C}$ & $\delta$ & No & Yes & No & 18 \\
\hline
\end{tabular}

Notes: "Meth" is the method used (QA $\rightarrow$ quasi-analytical, $3 \rightarrow 3$-body, $\mathrm{N} \rightarrow \mathrm{N}$-body), $N$ is the number of galaxies per group, "Soft" refers to the softening of the potential of the galaxy-galaxy interaction (Fix $\rightarrow$ fixed, $r \rightarrow$ scales as the galaxy radius, $r_{1 / 2} \rightarrow$ scales as the galaxy half-mass radius), "IGB" indicates the presence of an intergalactic background of dark matter, "ICs" refers to the initial conditions (V $\rightarrow$ Virialized, $\mathrm{C} \rightarrow$ Collapsing, $\mathrm{E} \rightarrow$ Expanding, and Circ $\rightarrow$ Circular orbits), "IMF" refers to the initial mass function ( $\delta \rightarrow$ equal masses, $\mathrm{S} \rightarrow$ a Schechter (1976) function, and $\mathrm{O} \rightarrow$ something else), "DF" refers to dynamical friction of galaxies against the dark matter background, "Merg" to the presence of a merger criterion, while "Tides" indicates the type of tidal interactions included $(\mathrm{C} \rightarrow$ collisional tides, IGB $\rightarrow$ IGB tides, and finally $\mathcal{N}$ is the number of simulations run. Moreover, the symbol "NR" indicates that the entry is not required for the type of simulations in consideration.

\section{Results}

I now concentrate on the self-consistent studies of groups listed in Table 2, and the two explicit-physics studies of Table 3 that correspond to groups (Roos \& Norman 1979; Mamon 1987). Qualitatively, the main result is rapid merging of galaxies leading to group coalescence. This is agreed upon by all 7 studies. How fast does this merging take on average? In Table 4 below, I compare the different studies, by evaluating for each the mean time for one merger, averaging over the time for 4 mergers to occur, or the full simulation time if 
shorter. These merger times are expressed in terms of the half-mass free-fall time defined as $t_{\mathrm{ff}}=(\pi / 2)\left(R_{h}^{3} / G M\right)^{1 / 2}$, where $R_{h}$ is the half-mass radius of the group at maximum expansion. I assume that $R_{h}$ is twice the half-mass radius of virialized groups. This estimator has the advantage of being independent of the state of evolution of the group and is more robust than the standard free-fall time often used.

Table 4: Merging times compared

\begin{tabular}{|c|c|c|c|c|c|c|c|c|c|}
\hline Author(s) & Run & $N$ & $n$ & IGB & ICs & $r_{h} / R_{h}$ & $t_{\mathrm{ff}}$ & $t_{m}$ & $t_{m} / t_{\mathrm{ff}}$ \\
\hline Roos \& Norman (79) & 1 & 10 & 1 & 0. & Vir & 0.034 & 11.3 & 6.6 & 0.59 \\
\hline Carnevali et al. (81) & $\begin{array}{l}26 \\
28 \\
29\end{array}$ & $\begin{array}{l}10 \\
20 \\
20\end{array}$ & $\begin{array}{l}20 \\
20 \\
20\end{array}$ & $\begin{array}{l}0 . \\
0 . \\
0 .\end{array}$ & $\begin{array}{l}\text { Vir } \\
\text { Vir } \\
\text { Vir }\end{array}$ & $\begin{array}{l}0.1 \\
0.05 \\
0.1\end{array}$ & $\begin{array}{l}3.3 \\
2.5 \\
2.7\end{array}$ & $\begin{array}{l}1.6 \\
1.25 \\
1.1\end{array}$ & $\begin{array}{l}0.49 \\
0.49 \\
0.41\end{array}$ \\
\hline Cavaliere et al. (83) & $12 B$ & 10 & 20 & 0.5 & Vir & $0.1 ?$ & 1.7 & 1.0 & 0.59 \\
\hline Barnes (85) & $\begin{array}{l}\text { A } \\
\text { B } \\
\text { C } \\
\text { D } \\
\text { E } \\
\mathbf{F} \\
\mathbf{G} \\
\mathbf{H} \\
\mathbf{I} \\
\mathbf{J} \\
\mathbf{K} \\
\mathbf{L}\end{array}$ & $\begin{array}{r}5 \\
5 \\
5 \\
5 \\
5 \\
5 \\
10 \\
5 \\
5 \\
5 \\
5 \\
5\end{array}$ & $\begin{array}{r}300 \\
300 \\
300 \\
300 \\
150 \\
150 \\
100 \\
150 \\
150 \\
75 \\
75 \\
75\end{array}$ & $\begin{array}{l}0 . \\
0 . \\
0 . \\
0 . \\
0.5 \\
0.5 \\
0.5 \\
0.5 \\
0.5 \\
0.75 \\
0.75 \\
0.75\end{array}$ & $\begin{array}{l}\text { Vir } \\
\text { Vir } \\
\text { Coll } \\
\text { Exp } \\
\text { Vir } \\
\text { Vir } \\
\text { Vir } \\
\text { Coll } \\
\text { Exp } \\
\text { Vir } \\
\text { Coll } \\
\text { Exp }\end{array}$ & $\begin{array}{l}0.1 ? \\
0.1 ? \\
0.1 ? \\
0.1 ? \\
0.1 ? \\
0.1 ? \\
0.1 ? \\
0.1 ? \\
0.1 ? \\
0.1 ? \\
0.1 ? \\
0.1 ?\end{array}$ & $\begin{array}{l}0.52 \\
0.28 \\
0.52 \\
0.52 \\
0.79 \\
0.52 \\
1.04 \\
0.79 \\
0.79 \\
1.04 \\
1.04 \\
1.04\end{array}$ & $\begin{array}{l}0.7 \\
0.6 \\
0.6 \\
1.0 \\
1.5 \\
2.0 \\
1.5 \\
1.0 \\
1.0 \\
1.6 \\
1.8 \\
1.5\end{array}$ & $\begin{array}{l}1.4 \\
2.1 \\
1.2 \\
1.9 \\
1.9 \\
3.9 \\
1.4 \\
1.3 \\
1.3 \\
1.5 \\
1.7 \\
1.4\end{array}$ \\
\hline Ishizawa (86) & $\begin{array}{l}\text { A } \\
\text { B } \\
\text { C } \\
\text { D } \\
\mathbf{E} \\
\mathbf{F}\end{array}$ & $\begin{array}{l}10 \\
10 \\
10 \\
10 \\
10 \\
10\end{array}$ & $\begin{array}{l}100 \\
100 \\
100 \\
100 \\
100 \\
100\end{array}$ & $\begin{array}{l}0 . \\
0 . \\
0 . \\
0 . \\
0 . \\
0 .\end{array}$ & $\begin{array}{l}\text { Coll } \\
\text { Coll } \\
\text { Coll } \\
\text { Coll } \\
\text { Coll } \\
\text { Coll }\end{array}$ & $\begin{array}{l}0.055 \\
0.026 \\
0.058 \\
0.052 \\
0.052 \\
0.052\end{array}$ & $\begin{array}{r}10.6 \\
31.6 \\
6.8 \\
11.5 \\
14.2 \\
14.6\end{array}$ & $\begin{array}{l}2.0 \\
7.5 \\
1.75 \\
2.75 \\
4.25 \\
4.25\end{array}$ & $\begin{array}{l}0.19 \\
0.24 \\
0.26 \\
0.24 \\
0.30 \\
0.29\end{array}$ \\
\hline Mamon (87) & $\begin{array}{l}\text { Dense } \\
\text { Dense } \\
\text { Dense } \\
\text { Dense } \\
\text { Loose } \\
\text { Loose } \\
\text { Loose } \\
\text { Loose }\end{array}$ & $\begin{array}{r}8 \\
20 \\
8 \\
20 \\
8 \\
20 \\
8 \\
20\end{array}$ & $\begin{array}{l}1 \\
1 \\
1 \\
1 \\
1 \\
1 \\
1 \\
1\end{array}$ & $\begin{array}{l}0.1 \\
0.1 \\
0.75 \\
0.75 \\
0.1 \\
0.1 \\
0.75 \\
0.75\end{array}$ & $\begin{array}{l}\text { Vir } \\
\text { Vir } \\
\text { Vir } \\
\text { Vir } \\
\text { Vir } \\
\text { Vir } \\
\text { Vir } \\
\text { Vir }\end{array}$ & $\begin{array}{l}0.39 \\
0.35 \\
0.1 \\
0.1 \\
0.04 \\
0.04 \\
0.015 \\
0.015\end{array}$ & $\begin{array}{l}0.35 \\
0.39 \\
0.28 \\
0.28 \\
8.6 \\
8.6 \\
3.9 \\
3.9\end{array}$ & $\begin{array}{l}0.125 \\
0.125 \\
0.75 \\
0.5 \\
6.67 \\
3.33 \\
10 . \\
6.67\end{array}$ & $\begin{array}{l}0.32 \\
0.32 \\
2.7 \\
1.8 \\
0.77 \\
0.39 \\
2.5 \\
1.7\end{array}$ \\
\hline Barnes (89) & & 6 & $8 k-16 k$ & 0. & Vir & 0.19 & 1.61 & 3.5 & 2.2 \\
\hline
\end{tabular}

NOTES: $N$ is the number of galaxies per group, $n$ is the number of particles per galaxy, "IGB" indicates the fraction of the group mass in an intergalactic background of dark matter, "ICs" refers to the initial conditions (Vir $\rightarrow$ Virialized, Coll $\rightarrow$ Collapsing, Exp $\rightarrow$ Expanding), $r_{h}$ and $R_{h}$ are the initial galaxy and group half-mass radii, respectively, $t_{\mathrm{ff}}$ is the half-mass free- 
fall time, and $t_{m}$ is the mean time between mergers (both are given in the units used in the study in consideraton). Note that Ishizawa's run A was presented in Ishizawa et al. (1983) and refers to an initial shell-like distribution of galaxies, his run $\mathrm{C}$ starting in a disk-like distribution, while his runs $\mathrm{D}$ to $\mathrm{F}$ are quite similar. The merging times of Barnes (1985) are based upon one to four runs, while Mamon's are based upon 50 and 10 runs for his groups of 8 and 20 galaxies, respectively.

From Table 4 on sees that collapsing groups merge at roughly the same rate as virialized groups. As an example, run $\mathrm{F}$ of Ishizawa, has a virial ratio of $2 T_{\text {rand }} /(-W)=0.5$ compared to his runs $B, D$, and $E$ for which this ratio is zero, and it's merging time is comparable with that of the other three runs. This is also well illustrated in the results of Barnes (1985). Ditto for expanding groups. Carnevali et al.'s results indicate little dependence of $t_{m}$ on group membership. But Barnes' run G shows a longer merging time, while Mamon's results show a contrary trend. One would expect that the normalized merging time should be much shorter in dense groups compared to loose ones. This is evident in Mamon's simulations of groups of 8 galaxies with individual halos, but not so in groups where the dark matter is mainly in the IGB. Perhaps this means that in the former case, where merging is "direct", the merger cross-sections are important, but that in the second case this is less so because dynamical friction against the IGB really sets the merging rates. This hypothesis is confirmed in Mamon (1987), where the merging rates in the groups with significant IGB are insensitive to the adopted merger cross-sections. The importance of the IGB has little direct effect according to Barnes (1985), but has a strong effect in Mamon (1987), who found that high $\mathrm{M} / \mathrm{L}$ groups with the same merger cross-sections have much longer merging times.

Are the normalized values of the merging time obtained in the different studies comparable? Consider first the runs with negligible mass in the IGB and starting from virialized initial conditions. Here, Mamon's merging times agree quite well with those of Roos \& Norman and Carnevali et al., while those of Barnes $(1985,1989)$ are roughly three to four times longer. Considering now virialized groups with half or more of their mass in an IGB, Cavaliere et al.'s merging time is roughly four times shorter than those of Barnes (1985) and Mamon. Now considering collapsing groups with no IGB, Ishizawa's merging times are typically five times shorter than those of Barnes (1985). These discrepancies are best explained as follows. Among self-consistent studies, those with the largest number of particles per galaxy are most reliable, so I prefer to emphasize the results of Ishizawa, Barnes (1985) and especially Barnes (1989). The departures of these studies from the other ones indicate that, for a negligible IGB, the merging times are longer than expected from two-body collisions, because the other galaxies in a group often come in and pump energy into the merging pair. This is well illustrated in the movie of Barnes (1989). Hence explicit-physics computations ought to use smaller merger cross-sections compared to those given in the litterature of two-galaxy collisions. For groups with a dominant IGB, this effect is not seen since the merging rates are less sensitive to the merging process itself (see above). The discrepancy between Barnes 1985 and Ishizawa may perhaps be explained by the combination of two factors: first in a collapsing group the merger rate ought to be proportional to the number of galaxies in the group, as they all collapse together. Second, Barnes uses three times as many particles and can probably thus distinguish the merging cores longer than Ishizawa.

One should note here that any discrepancy of a factor two is not significant as Mamon finds a standard deviation of 0.3 in the $\log$ of his evolution times. But how long can a lucky 
dense group survive merging? Mamon's simulations show that one can remain with 4 galaxies (starting with 8 to 20 ) after a Hubble time (only roughly $5 \%$ of the time and only for certain parameters). However, the surviving groups are always very "evolved" in the sense that their range of luminosities is greater than allowed by Hickson's HCG selection criteria. Therefore, if HCGs are dense and bound systems they cannot be the remnants of early very dense density pertubations, but must have formed instead more recently, presumably by two-body interactions within looser systems. This issue is addressed by Mamon (1987) who looked for examples of rapid succession of mergers in his simulated loose groups and found one possible case out of 50 simulations of loose groups of 8 galaxies and a dominant IGB, and argues that such events are insignificant in the loose groups of 8 galaxies with individual halos. On the other hand, Mamon found a probability of 5 to $25 \%$ of observing a a simulated loose group and finding a projected configuration that satisfied Hickson's HCG criteria. Recently, Walke \& Mamon (1989) have argued that these numbers would be reduced to roughly one percent had Mamon adopted more realistic larger sizes for his loose groups. Finally, of these compact projected configurations seen by Mamon, roughly 15 and $45 \%$ of these are in fact catsed by 3D chance configurations (transient cores).

What physical mechanisms are dominant? This question is well addressed by the explicitphysics simulations of Mamon. Merging turns out to be the dominant mechanism that causes a dense group to lose its HCG appearence, but while merging is "direct" when the galaxies have large merging cross-sections due to their individual halos of dark matter, the evolution of dense groups of galaxies orbiting within a dominant IGB is different. Here, dynamical friction of the galaxies against the IGB forces the galaxies to meet at the group center where they cannot avoid merging, but this takes much more time (see Table 4). When Mamon turned off dynamical friction, the first type of groups still merged at the same rate, while the second type barely did so. Contrary to the situation in globular clusters, evaporation is insignificant in groups of galaxies, simply because the potential wells of galaxies are not deep enough. In dense groups, tides turn out to be important in limiting the sizes of the dominant cannibal galaxies, and the IGB tides are much more effective than the collisional tides. Beyond a luminosity of roughly $4 L_{*}$, a cannibal galaxy cannot hold on to its victims and returns them (in digested form!) to the IGB. Tides and the paucity of galaxies thus prevent any real merging instability as described by Ostriker \& Hausman (1977): This is checked from the plots of mergers versus time of Mamon (1987), from which the merger rates start out constant in dense groups with individual halos, but vary as $1 / t$ in dense groups with the dark matter in the IGB.

In the end one gets one giant cannibal, which appears very much like a giant elliptical, as well illustrated by the $r^{1 / 4}$ surface brightness distribution found by Barnes (1989), who can also just barely distinguish shells. Thus the outcome of multiple mergers in dense groups is naturally quite similar to that of simple mergers of colliding pairs (e.g., Barnes 1988).

After short periods of time, dense groups show strong signs of dynamical interaction, as well illustrated by the statistical averages over Mamon's explicit-physics simulations. For example, if the dark matter is mainly in the IGB, then luminosity segregation sets in quite rapidly (due to more rapid orbital decay by dynamical friction for high-mass galaxies). Moreover, wherever the dark matter was placed, the statistics of the bright-end of the galaxy luminosity functions, showed signatures of significant merging also develop very rapidly. 


\section{Conclusion}

In summary, dense groups of galaxies have been simulated in seven different studies, with reasonably consistent results. These groups show rapid merging, although this is mainly due to their short dynamical times. It is very unlikely that more than a few percent of dense groups can survive for over a Hubble time, and the few that do bare little resemblence to Hickson's compact groups. Whether abundant in nature or not, dense groups provide a fascinating laboratory to study galaxy interactions pushed to the extreme, and one learns that tidal processes manage to slow down the merging process, and that orbital decay by dynamical friction of galaxies against an intergalactic background of dark matter is significant in speeding it up. With the advent of increasingly powerful computing facilities, time will come when the statistical studies done with explicit-physics studies will be achievable with self-consistent studies. Along these lines is the work by Borne and Levison (in preparation) who are using a restricted 3-body code to perform large numbers of dense group simulations that ought to be more reliable than Mamon's explicit-physics study. talk.

I gratefully acknowledge Josh Barnes for providing on very short notice a movie for my

\section{References}

Aarseth, S.J., and Fall, S.M. 1980, Ap. J., 236, 43.

Barnes, J. 1985, M.N.R.A.S., 215, 517.

Barnes, J. 1988, Ap. J., 331, 699.

Barnes, J. 1989, Nature, 338, 123.

Carnevali, P., Cavaliere, A., and Santangelo, P. 1981, Ap. J., 249, 449.

Cavaliere, A., Santangelo, P., Tarquini, G., and Vittorio, N. 1982, in Clustering in the Universe, ed. D. Gerbal and A. Mazure (Gif-sur-Yvette: Editions Frontières), p. 25.

Cooper, R.G., and Miller, R.H. 1981, Ap. J., 254, 16.

Dekel, A., Lecar, M., and Shaham, J. 1980, Ap. J., 241, 946.

Hickson, P. 1982, Ap. J., 255, 382.

Ishizawa, T., Matsumoto, R., Tajima, T., Kageyama, H., and Sakai, H. 1983, P.A.S.J., 35, 61.

Ishizawa, T. 1986, Ap. Sp. Sci., 119, 221.

Jones, B.J.T., and Efstathiou, G. 1979, M.N.R.A.S., 189, 27.

Malumuth, E.M., and Richstone, D.O. 1984, Ap. J., 276, 413.

Mamon, G.A. 1985, Ph.D. thesis, Princeton University.

Mamon, G.A. 1986, Ap. J., 307, 426.

Mamon, G.A. 1987, Ap. J., 321, 622.

Merritt, D. 1983, Ap. J., 264, 24.

Miller, G.E. 1983, Ap. J., 268, 495.

Navarro, J.F., Mosconi, M.B., and Lambas, D.G. 1987, M.N.R.A.S., 228, 501.

Ostriker, J.P., and Hausman, M. 1977, Ap. J. (Letters), 176, L51.

Richstone, D.O. 1976, Ap. J., 204, 642.

Richstone, D.O., and Malumuth, E.M. 1983, Ap. J., 268, 30.

Roos, N. 1981, Astr. Ap., 95, 349.

Roos, N., and Aarseth, S.J. 1982, Astr. Ap., 114, 41.

Roos, N., and Norman, C.A. 1979, Astr. Ap., 76, 75.

Rose, J.A. 1979, Ap. J., 231, 10.

Saarinen, S., and Valtonen, M.J. 1985, Astr. Ap., 153, 130.

Schneider, D., and Gunn, J.E. 1982, Ap. J., 263, 14.

Tully, R.B. 1987, Ap. J., 321, 280.

Walke, D.G., and Mamon, G.A. 1989, Astr. Ap., 295, 291. 


\section{DISCUSSION}

Rennicutt: 1. In a typical group merger what fraction of the stars are ejected from the system? 2. The end product of Barnes' simulation looked very much like a field CD galaxy. Are the observed space densities and luminosity functions of isolated $\mathrm{CD}$ and $\mathrm{E}$ galaxies consistent with the compact group densities and merger timescales?

Mamon: 1. I can't say for dense groups, but presumably, the fraction of ejected stars should be the same as in isolated mergers, i.e., 5-108 depending on who you ask. 2. Assuming a constant rate of compact group coalescence, I found that between 5 and 408 of all giant ellipticals outside of clusters ought to be the result of not one merger, but at least three consecutive mergers. However, it seems that dense groups must form within loose groups (and on fact I just showed that all nearby compact groups are situated within looser systems) - Hence the end product in this case could resemble more CD poor (MKW) clusters.

Hickson: I understand that in Barnes' simulation only about half the mass was in dark halos and that none of this was in a common envelope. This seems inconsistent with the high mass-to-light ratios that are observed in compact groups. What would be the effect of increasing the mass in dark halos and in a common envelope?

Mamon: I agree with you that Barnes has not put enough dark matter in his simulations. If you put significant additional dark matter in individual halos, then these will have larger cross-sections and the merging times will be decreased. If instead you put this additional dark matter in a diffuse intergalactic background there will be competition between two effects. On one hand, the galaxies will experience stronger dynamical friction, their orbits will decay faster and thus merge faster. On the other hand, the background will exert stronger tides on the galaxies, thus reducing the merger cross-sections and thus the merger rates. The level to which one should increase the dark matter background (assuming that is where you put it), I think, is such that the first effect should at least be comparable to the second.

H. Roos: (comment on Hickson's question) I think that increasing the $M / L$ ratio of groups by increasing the amount of dark matter in the background will also decrease the merging time due to dynamical friction, although maybe not so strong as in the case where you increase the halo mass of the galaxies. 


\section{DISCUSSION}

Appleton: Could you say something about the evolution of the dwarf galaxy population during the "merging instability" stage in compact group simulations? Do you think that the lower end of the group luminosity function might be a useful indicator of what is going on in such groups (i.e., how dynamically evolved the system is).

Mamon: The low mass galaxies, being smaller, merge less efficiently, and when they do, they presumably bump into massive galaxies. But whereas I've studied the evolution of the bright end of the luminosity functions of the simulated dense groups, I've not really paid any attention to what goes on at the faint end.

Zasov: Could you tell us a little more about the merger itself? Is it consistent with the Faber-Jackson or Tully-Fisher relations? What's the fraction of dark matter inside of the "optical" boundaries? What galaxy does it look like?

Mamon: The detailed simulations by Barnes show that the merger remnents look like the merger remnents of isolated pairs of colliding galaxies which themselves resemble elliptical galaxies. Presumably the outer regions of the halos heat up during the encounters and settle mainly beyond the "optical" boundary. In my own simulations the original Faber-Jackson relation was not too affected by mergers.

Fridman: Do you think that the process of an artificial satellite falling on the Earth because of an atmospheric friction can be considered "merging instability"?

Mamon: It all depends on the range of masses involved in the merging. Merging usually involves a massive galaxy being swallowed by an even more massive one - the typical ratio of merging masses is about 3 . So a typical merger increases the main galaxy's mass by roughly one-third and its cross-section by roughly two-thirds. Granted, its not a drastic instability. Your example is in principle an instability too, although a very inefficient one! 